\title{
Impact of perinatal factors on continuous early monitoring of brain electrocortical activity in very preterm newborns by amplitude-integrated EEG
}

\author{
Giancarlo Natalucci ${ }^{1,2}$, Cornelia Hagmann ${ }^{1}$, Vera Bernet ${ }^{3}$, Hans-Ulrich Bucher ${ }^{1}$, Valentin Rousson ${ }^{4}$ and Beatrice Latal ${ }^{2}$
}

BACKGROUND: Amplitude-integrated electroencephalogram (aEEG) is increasingly used for neuromonitoring in preterms. We aimed to quantify the effects of gestational age (GA), postnatal age (PNA), and other perinatal factors on the development of aEEG early after birth in very preterm newborns with normal cerebral ultrasounds.

METHODS: Continuous aEEG was prospectively performed in 96 newborns (mean GA: 29.5 (range: 24.4-31.9) wk, birth weight 1,260 (580-2,120) g) during the first $96 \mathrm{~h}$ of life. aEEG tracings were qualitatively (maturity scores) and quantitatively (amplitudes) evaluated using preestablished criteria.

RESULTS: A significant increase in all aEEG measures was observed between day 1 and day 4 and for increasing GA ( $P$ $<0.001$ ). The effect of PNA on aEEG development was 6.4- to 11.3-fold higher than that of GA. In multivariate regression, GA and PNA were associated with increased qualitative and quantitative aEEG measures, whereas small-for-GA status was independently associated with increased maximum aEEG amplitude $(P=0.003)$. Morphine administration negatively affected all aEEG measures $(P<.05)$, and caffeine administration negatively affected qualitative aEEG measures $(P=0.02)$.

CONCLUSION: During the first few days after birth, aEEG activity in very preterm infants significantly develops and is strongly subjected to the effect of PNA. Perinatal factors may alter the early aEEG tracing and interfere with its interpretation.

$\mathrm{n}$ the last three decades, advances in perinatal medicine have led to a substantial increase in survival rate for preterm infants (1). As neurodevelopmental morbidity did not decrease in a corresponding order (2), the focus has shifted toward prevention and early detection of brain injury. One approach is through continuous monitoring of brain activity using amplitude-integrated electroencephalography (aEEG). In term infants with perinatal hypoxic-ischemic encephalopathy, aEEG background pattern characteristics and changes in cycling activity provide prognostic information on neurodevelopmental outcome (3). Recent studies demonstrated that early aEEG pattern can predict both short- and long-term outcome in the preterm $(4,5)$. Normal aEEG background pattern in preterm infants (6) differs, however, from that in the term infants (7), and interpretation of its tracing is problematic because it is strongly influenced by brain maturation $(6,7)$. With increasing gestational age (GA) and postnatal age (PNA), immature aEEG pattern and low-voltage activity decrease $(6,8)$. Additionally, medications, such as sedatives (9), frequently used in the neonatal intensive care unit (NICU) also influence aEEG activity, which makes aEEG interpretation in sick and immature infants particularly difficult. As aEEG is being increasingly used for clinical surveillance in many NICUs, clinicians face the challenges of interpreting preterm aEEG. However, it still remains to be elucidated how and under which conditions aEEG tracing evolves during the very early life period in this often critically ill patient population (10). Thus, our study investigates the evolution of aEEG tracing during the first $4 \mathrm{~d}$ of life in preterm infants with GA $<32$ wk without any brain injury on cranial ultrasound scan (cUS) and without any relevant hemodynamic disturbance. aEEG was measured repeatedly every $3 \mathrm{~h}$. The influence of GA and PNA on the course of aEEG activity is analyzed, adjusting for several, potentially confounding perinatal factors. We hypothesize that early aEEG tracing activity increases during the first few days of life and that its interpretation is affected by perinatal non-neurological factors.

\section{RESULTS}

\section{Study Subjects}

During the study period, of the 301 eligible newborns at the first day of life, 148 were prospectively monitored with aEEG and among them 96 were enrolled. Of the remaining 153 nonmonitored infants, 117 eligible infants could not be enrolled because of the limited capacity to simultaneously monitor brain activity of more than two infants in our division (Figure 1). The aEEG tracings of 96 newborns with a mean (range) GA of

\footnotetext{
The last two authors shared last authorship.

'Department of Neonatology, Zurich University Hospital, Zurich, Switzerland; ${ }^{2}$ Child Development Centre, Zurich University Children's Hospital, Zurich, Switzerland;

${ }^{3}$ Department of Pediatric Intensive Care and Neonatology, Zurich University Children's Hospital, Zurich, Switzerland; ${ }^{4}$ Centre Universitaire Hospitalier Vaudois and University of Lausanne, Statistical Unit, Institute of Social and Preventive Medicine, Lausanne, Switzerland. Correspondence: Giancarlo Natalucci (giancarlo.natalucci@usz.ch) 


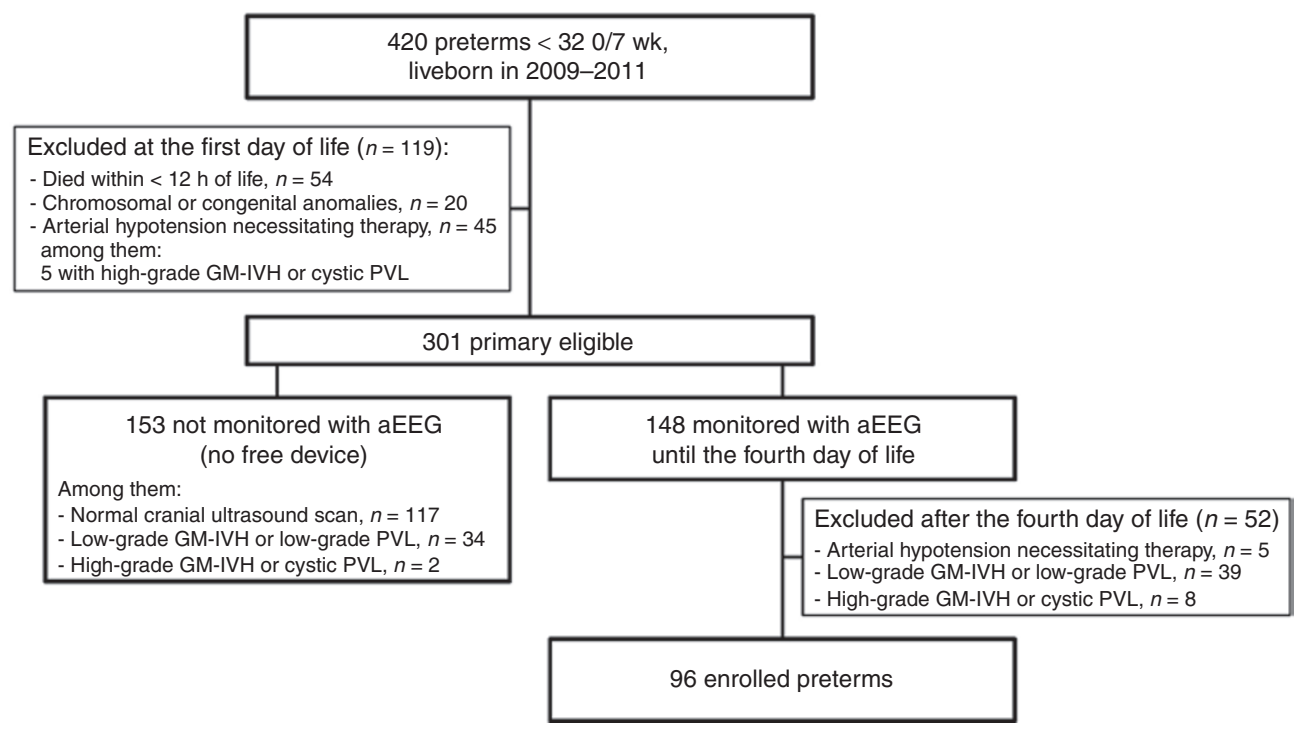

Figure 1. Diagram flow of the study participants. Germinal matrix-intraventricular hemorrhage (GM-IVH) graded according to ref. 30: low-grade GM-IVH, grade I and II GM-IVH; high-grade GM-IVH, grade III GM-IVH and parenchymal hemorrhagic infarction. Periventricular leukomalacia (PVL) graded according to ref. 31: low-grade PVL, non-cystic PVL, grade I; cystic PVL, grades II, III, and IV. aEEG, amplitude-integrated electroencephalogram.

29.5 (24.4-31.9) wk and a mean birth weight of 1,260 (580$2,120) \mathrm{g}$ were evaluated. The recordings started at a mean (range) age of $13.0(1-21) \mathrm{h}$ postpartum and were continuously performed until a mean (range) age of $90.0(72-96) \mathrm{h}$ after birth. Infants who received antenatal corticosteroids, mechanical ventilation, and morphine, caffeine, or indomethacin had significantly lower GA compared with those who did not (Mann-Whitney Test, all $P<0.001$ ). No infant suffered from seizures. The perinatal baseline characteristics of the study infants listed in Table 1 did not differ from that of the eligible infants but from that of the nonmonitored infants $\left(\chi^{2}\right.$ and Mann-Whitney test, all $P>0.1$ ).

\section{Interrater Agreement}

Cohen's $\kappa(95 \%$ confidence interval) for interrater agreement was $0.79(0.75-0.82)$ for the "maturity total score" and 0.60 (0.52-0.66) for the "cycling subscore," respectively.

\section{Univariate Effects of GA and PNA on aEEG Tracing}

In the univariate analysis of the mixed model (Table 2), GA was positively and significantly associated with all aEEG measures. PNA was also positively, but even stronger associated with all aEEG measures. Figure 2 shows an example of an aEEG development of a study subject from day 1 to day 3 of life with the corresponding aEEG measures. The slope for the PNA was 6.4 (total maturity score) to 11.3 times (maximum aEEG amplitude) steeper than for the GA. Figure $3 a-c$ illustrates these effects for the total maturity score and the maximum and minimum aEEG amplitude.

\section{Univariate Effects of Perinatal Factors on aEEG Tracing}

Univariate regression analyses of the linear mixed model revealed (Table 2) that (i) infants born SGA displayed
Table 1. Perinatal variables of the study subjects

\begin{tabular}{|c|c|}
\hline Perinatal variables & $n=96$ \\
\hline Gestational age (wk), m (range) & $29.5(24.4-31.9)$ \\
\hline Birth weight (g), m (range) & $1,260(580-2,120)$ \\
\hline $\mathrm{SGA}, n(\%)$ & $14(16)$ \\
\hline Male sex, $n(\%)$ & $53(55)$ \\
\hline Preeclampsia, $n(\%)$ & $23(24)$ \\
\hline Chorioamnionitis, $n(\%)$ & $18(19)$ \\
\hline Antenatal steroids & $72(75)$ \\
\hline Cesarean section, $n(\%)$ & $85(88)$ \\
\hline Arterial cord pH, m (SD) & $7.30(0.10)$ \\
\hline 5-min Apgar, m (SD) & $6.8(2.0)$ \\
\hline SNAPPE-II, M (IQR) & $18(5-28)$ \\
\hline Respiratory distress, $n$ (\%) & $88(92)$ \\
\hline Mechanical ventilation, $n(\%)$ & $34(35)$ \\
\hline Days on mechanical ventilation, M (IQR) & $1(1-4)$ \\
\hline Surfactant, $n(\%)$ & $33(34)$ \\
\hline Early onset sepsis, $n(\%)$ & $3(3)$ \\
\hline Hypoglycemia, $n(\%)$ & $2(2)$ \\
\hline Morphine ${ }^{\mathrm{a}}$ during aEEG, $n(\%)$ & $15(17)$ \\
\hline Caffeine $^{\mathrm{b}}$ during aEEG, $n(\%)$ & $21(22)$ \\
\hline Indomethacin` during aEEG, $n$ (\%) & $28(29)$ \\
\hline
\end{tabular}

aEEG, amplitude-integrated electroencephalogram; hypoglycemia, blood glucose level <2.5mmol//; IQR, interquartile range; $\mathrm{m}$, mean; $\mathrm{M}$, median; SGA, small for gestational age, defined as the birth weight $<10$ centile; SNAPPE-II, score for neonatal acute physiology perinatal extension-II (ref. 20).

aMedian (range) dose of 6 (6-12) $\mathrm{mg} / \mathrm{kg} / \mathrm{h}$, during median (range) of $43(12-60) \mathrm{h}$ of aEEG monitoring. bFirst bolus of $20 \mathrm{mg} / \mathrm{kg}$ from the median (range) $43 \mathrm{rd}$ (20th-64th) h of life and further daily administration of $5 \mathrm{mg} / \mathrm{kg} / \mathrm{d}$. cSix doses of $0.1 \mathrm{mg} / \mathrm{kg} / \mathrm{d}(n=$ 16) or three doses of $0.2 \mathrm{mg} / \mathrm{kg} / 12 \mathrm{~h}(n=12)$ from the $68(50-70) \mathrm{h}$ of life. Morphine and caffeine were administered intraarterially or intravenously, and indomethacin was administered intravenously. 
Table 2. Univariate effect of perinatal factors on aEEG measures

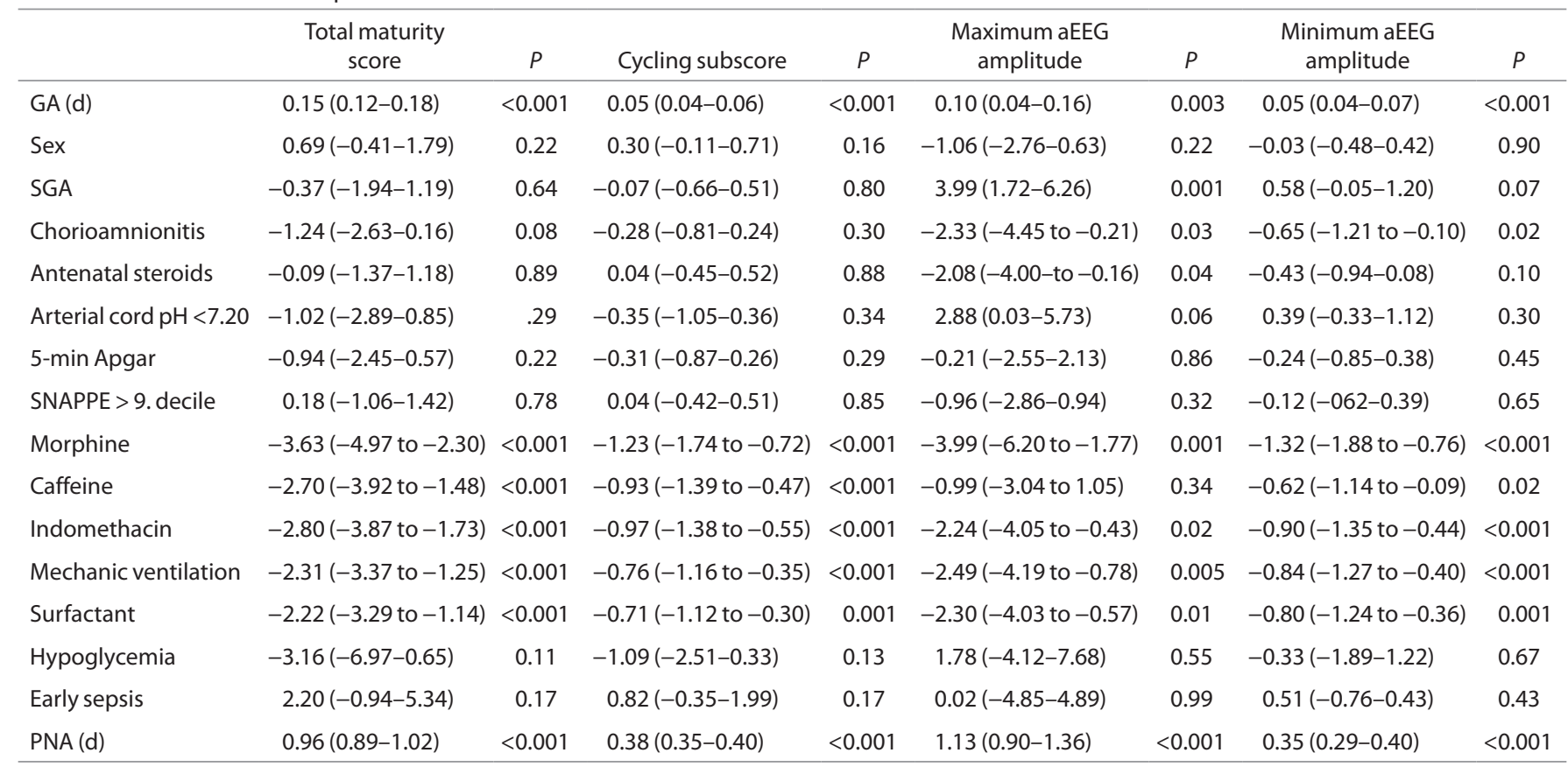

Slope coefficients with ( $95 \%$ confidence interval) are given.

aEEG, amplitude-integrated electroencephalogram; decile, highest decile for GA of the SNAPPE-II Edition (20); GA, gestational age; PNA, postnatal age, continuous data; SGA, small for GA, defined as a birth weight below the 10th percentile; SNAPPE-II, score for neonatal acute physiology perinatal extension-II.

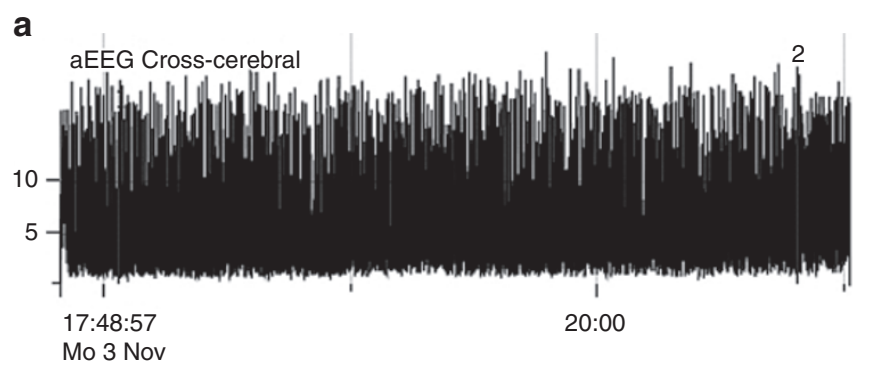

b

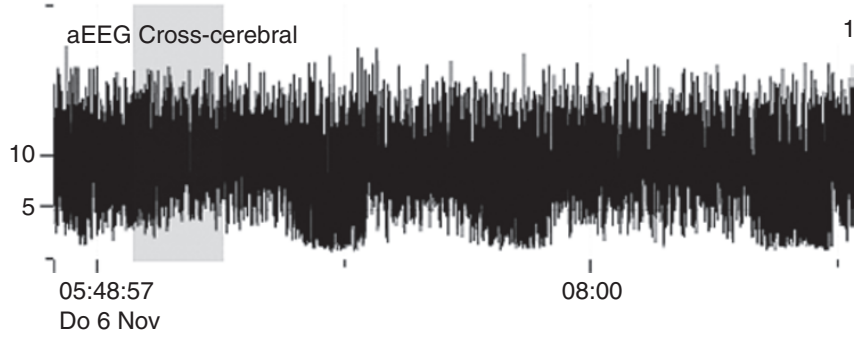

Figure 2. Amplitude-integrated electroencephalogram (aEEG) maturation in a study subject (gestational age: $30.0 \mathrm{wk}$ ) at (a) day 1 and (b) day 3 of life with the corresponding aEEG measures. (a) Day 1 (7-10 h after birth): maturity total score 1; cycling subscore 0 ; mean maximal aEEG amplitude $26.8 \mu \mathrm{V}$; and mean minimal aEEG amplitude $3.2 \mu \mathrm{V}$. (b) Day 3 (67-70 h after birth): maturity total score 8 ; cycling subscore 2; mean maximal aEEG amplitude $23.8 \mu \mathrm{V}$; and mean minimal aEEG amplitude 5.6 $\mu \mathrm{V}$.

significant higher maximum aEEG amplitude than those born appropriate for GA; (ii) chorioamnionitis and administration of antenatal steroids were associated with a decrease in maximum aEEG amplitude; and (iii) and the administration of morphine, caffeine, indomethacin, surfactant, and mechanical ventilation were associated with less-mature aEEG measures. aEEG parameters were not associated with the sex of the subjects or any of the following measures of peri- and postnatal adaptation (arterial cord pH, 5-min Apgar score, score for neonatal acute physiology perinatal extension-II (SNAPPE-II), early sepsis, and hypoglycemia).

\section{Multivariate Effects of GA, PNA, and Perinatal Factors on aEEG}

Multivariate regression analysis of the linear mixed model revealed (Table 3) that (i) GA (but the maximum aEEG amplitude) and PNA were still significantly associated with an increase in all aEEG measures; (ii) small-for-GA status was associated with increased maximum aEEG amplitude; and (iii) the administration of morphine and caffeine (but the "cycling subscore") was associated with a decrease in the qualitative aEEG measures (Figure 3).

\section{DISCUSSION}

This study describes changes of continuous aEEG tracings during the first 4 days of life in very preterm infants without cerebral injury on cUS. A significant increase in the aEEG activity during this early period of life was observed. Our findings are consistent with previous studies showing that qualitative (maturity scores) and quantitative (amplitudes) aEEG characteristics increase with advancing GA and PNA (8-15). According to our results, it seems that in very preterm infants, PNA is a more powerful drive for the development of aEEG measures than GA during this early period after birth, as observed previously by others over similar recording periods (13) and in serial recordings over longer observational periods (8) than in our study. The new analysis approach, the 

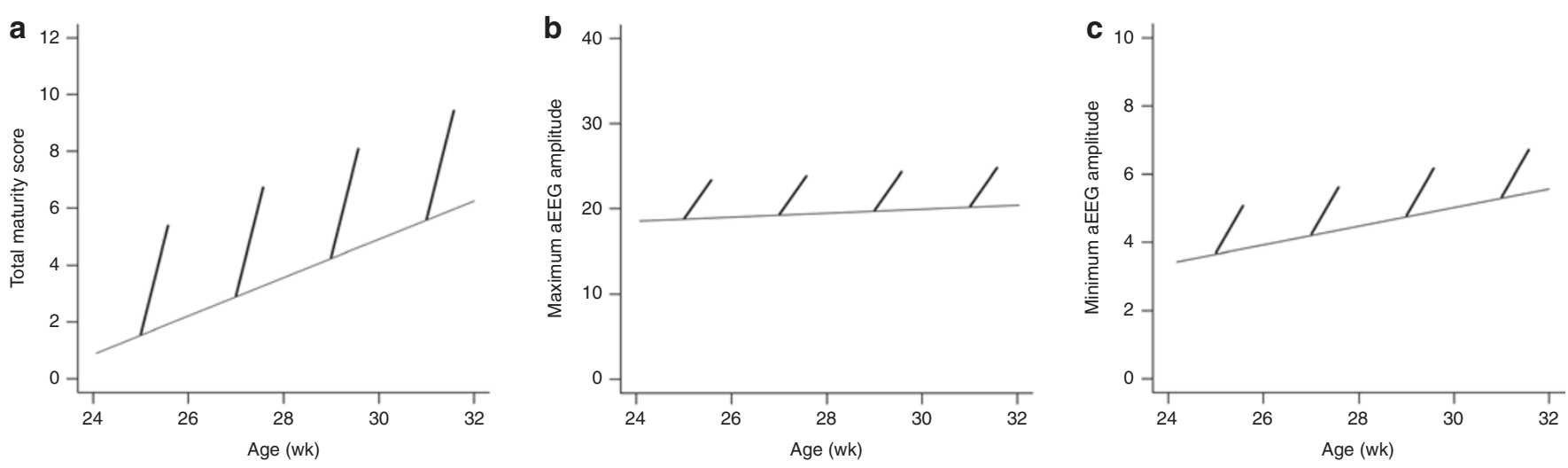

Figure 3. Effects on amplitude-integrated electroencephalogram (aEEG) tracing of gestational age (GA) and postnatal age (PNA) for (a) the "maturity total score," (b) the maximum, and (c) minimum aEEG amplitude measurements. The continuous gray line represents the expected aEEG at birth for a reference infant in function of her GA (expressed here in weeks), estimated from our linear mixed model. The black segments of line represent the expected aEEG during the first 4 days (i.e., the first half of a week) of life, estimated from our linear mixed model. These segments are different for each $\mathrm{GA}$ because the expected aEEG at birth depends on GA (shown here are segments at 25, 27, 29, and $31 \mathrm{wk}$ of GA). The slope of the GA is thus equal to the estimated GA effect, and the slope of the black segments is thus equal to the estimated PNA effect. The fact that the latter are steeper than the former illustrates the fact that the estimated PNA effect is higher than the estimated GA effect. Range of the four aEEG measures: total maturity score 0-12; cycling subscore: 0-4; maximum aEEG amplitude 4.5-36.0 $\mu \mathrm{V}$; and minimum aEEG amplitude 1.6-8.5 $\mu \mathrm{V}$. The results are corrected for sex, small for GA status, chorioamnionitis, antenatal steroids, 5-min Apgar, SNAPPE > 9. decile, administration of morphine, caffeine, and indomethacin, mechanical ventilation, and surfactant. SNAPPE-II, score for neonatal acute physiology perinatal extension-II.

Table 3. Multivariate effect of perinatal factors on aEEG measures

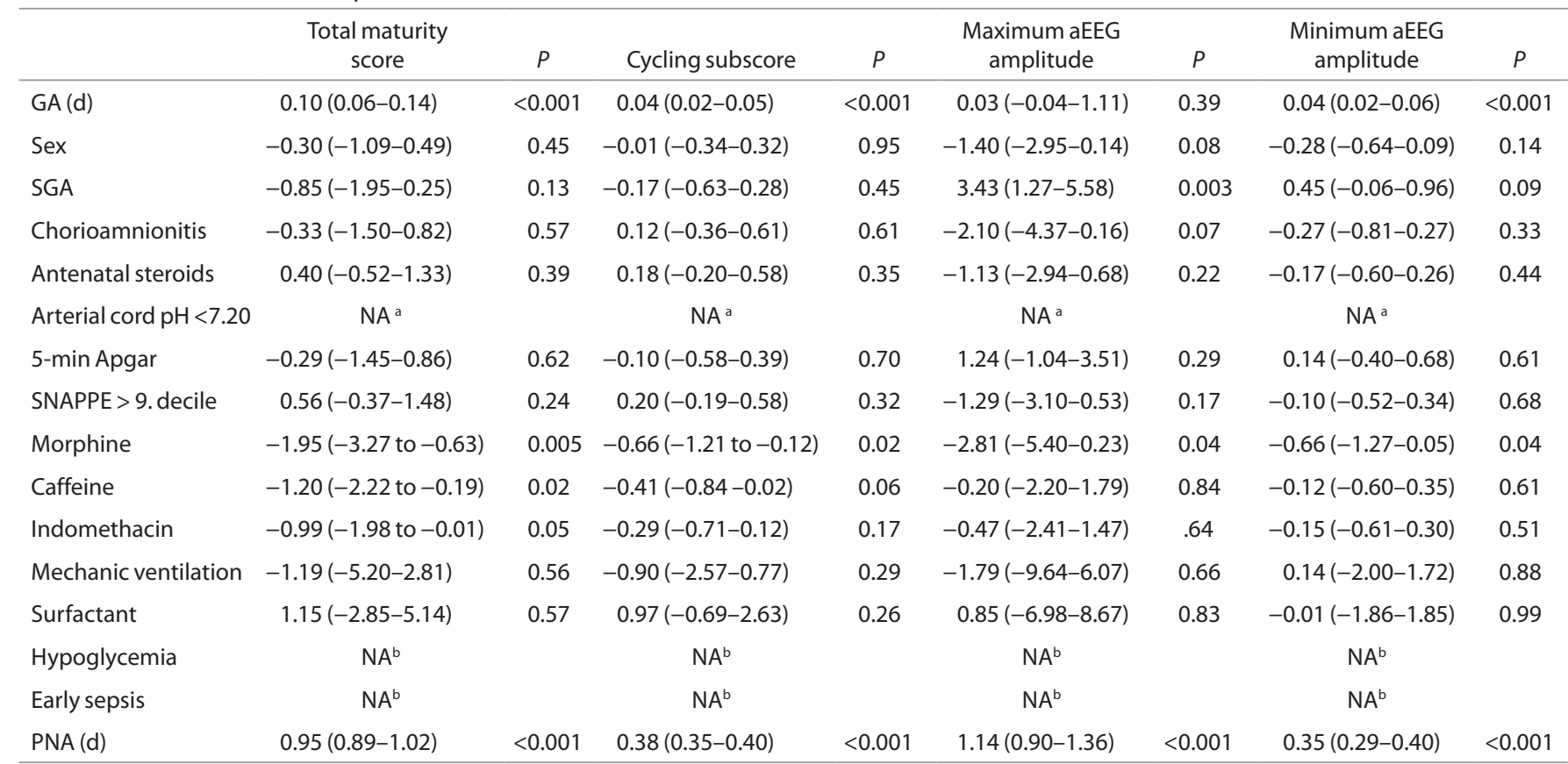

Slope coefficients with ( $95 \%$ confidence interval) are given.

aEEG, amplitude-integrated electroencephalogram; decile, highest decile for GA of the SNAPPE-II Edition (20); GA, gestational age; NA, not assessed; PNA, postnatal age, continuous data; SGA, small for GA, defined as a birth weight below the 10th percentile; SNAPPE-II, score for neonatal acute physiology perinatal extension-II Edition (20).

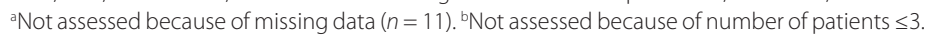

adjustment for several perinatal factors, and the large cohort size relative to the majority of studies on the same topic represent the strength of our study.

The observed increase of the electrocortical activity in preterm newborns immediately after birth may be the result of different processes. It may reflect an initial suppression following perinatal depression and consequent recover within $4 \mathrm{~d}$ (Figure 2). The lack of any association between arterial cord
$\mathrm{pH}$, Apgar or severity illness scores, and the aEEG measures in our cohort does not support this hypothesis. Alternatively, the steeper developmental slope for PNA than for GA of the aEEG measures may be explained by an adaptation of the immature brain to postnatal stress factors such as sensorial stimuli and NICU interventions. NICU interventions are effectively performed more frequently during the first few days after birth, when these infants were monitored than at a later 


\section{Articles $\mid$ Natalucciet al.}

age. However, as we did not prospectively record and quantify these stimuli (pain, noise, light etc.) in our study, we cannot prove that this hypothesis holds true. There is evidence that the exposure to standard recorded stressors in the NICU is associated with regional alteration in brain structure when measured by magnetic resonance imaging (16). Other studies showed that preterm birth itself can alter functional brain maturation resulting in neurophysiological dysmaturity, with NICU interventions being a potential causative factor $(17,18)$. Literature on neurosensory brain activity in preterm infants in relation to extrauterine life is inconsistent. The maturation of somatosensory pathways in preterm infants seems to be not altered in preterms without neurological deficit (19). The rapid decrease in latencies of the auditory (20) or visual (21) pathways, however, appears to be related to extrauterine factors rather than GA. EEG sleep studies, focusing on brain organization and maturation, reported both delayed and accelerated development of the cortical activity in healthy preterm infants (17), as well as the capacity of the immature brain to react differently to environment interventions (22).

This study shows that aside from GA and PNA, the administration of morphine and caffeine independently influenced the aEEG tracing, i.e., led to a less-mature background pattern and lower amplitude. Despite performing a multivariate analysis, controlling for GA and neonatal medical complications, it may be argued that newborns receiving these medications were more immature and sick, and thus, the underlying condition may have a greater impact on aEEG tracings than the interventions themselves.

We observed a suppressive effect of continuous morphine therapy on aEEG measures. This has not been reported in term cardiac infants (23), but it has already been demonstrated for preterms after morphine bolus application (24). According to multivariate analyses, the suppressive effect of morphine was only seen for the qualitative aEEG but not for the quantitative measures.

In our study, caffeine was associated with less-mature aEEG measures. This is in contrast with the findings of a study in which aEEG amplitudes and periods of continuity increased significantly in the immediate $2 \mathrm{~h}$ after caffeine administration (25). However, it remains unclear whether the effect on aEEG arises from the apneas and the underlying cerebral immaturity or from the caffeine administration. As we did not record the frequency of apneas in our sample, we are not able to draw a final conclusion.

The higher maximum aEEG amplitude observed in smallfor-GA compared with appropriate-for-GA preterms could reflect a less-synchronous EEG activity pattern and possibly a less-mature ability to adapt to the extrauterine environment. EEG dysmature or disorganized pattern of activity has been already observed by others in intrauterine growth retarded preterms (26).

Our observation of an association between chorioamnionitis and lower maximum and minimum aEEG amplitudes in the univariate analysis is in agreement with the observation that high levels of tumor necrosis factor- $\alpha$ in preterms are associated with EEG depression (27). Similarly, recent data from animal studies suggest that subclinical exposure of preterm sheep to low-dose endotoxin can impair EEG maturation during the first $10 \mathrm{~d}$ of life, associated with neural inflammation (28).

The main limitation of this work is the rather small sample for the number of cofactors analyzed in a multivariate analysis. As only two aEEG devices were available in our unit, it was impossible to enroll the majority of newborns in our NICU. Recruitment bias, however, seems to be rather improbable considering the similar perinatal baseline characteristics of recruited vs. nonrecruited newborns. Furthermore, aEEG raters were blinded to the GA but not to the PNA of the subjects as they scored full aEEG tracings. This fact might have biased the visual assessment of the aEEG, potentially overestimating the postnatal increase of the scores. On the other hand, as our accuracy of GA estimate is not precise to $1 \mathrm{~d}$, the estimation of the true GA effect on aEEG measures could have been attenuated. Finally, our interrater agreement for the visual aEEG assessment was of moderate degree, which may have decreased the power of the analysis of the course of the aEEG tracings over time. No bias should have implied the analysis of the difference between groups, however, as the same rater was used for both groups.

In conclusion, this study shows that in very preterm newborns, aEEG activity undergoes a significant maturational acceleration during the first few days of life. PNA and GA have the strongest influence on the development of the aEEG tracing; however, also non-neurologic clinical conditions and the administration of medications such as morphine and caffeine may relevantly alter the aEEG tracing and confound its interpretation. Knowledge on the impact of potential aEEG signal confounders is needed in order to improve the interpretation of aEEG tracings and thereby establish this neuromonitoring tool also for preterm born infants.

\section{METHODS}

The institutional ethics boards of the University Children's Hospital Zurich and of the Canton of Zurich approved the study protocol. Written informed consent was obtained from the parents or primary caretakers.

\section{Subjects}

In this prospective study, inborn infants with GA $<32 \mathrm{wk}$ admitted to the NICU of the neonatology division of the Zurich University Hospital between January 2009 and December 2011 were eligible. Exclusion criteria included chromosomal or congenital anomalies; any abnormality observed on cUS at any time after birth until discharge from the neonatology division; inborn errors of metabolism; or arterial hypotension (29), necessitating therapy with inotropes or vasopressors during aEEG monitoring. An abnormality on cUS was defined as any grade of germinal matrix-intraventricular hemorrhage according to Volpe (30), any grade of periventricular leukomalacia according to de Vries et al. (31), and ventriculomegaly according to Levene et al. (32). The NICU neonatologists performed the cUS, the findings were regularly reviewed within the board (including cUS specialists), and if disagreement was present, the rating was discussed and agreed upon. GA was assigned according to the best obstetrical estimate based on the last menstrual cycle and prenatal ultrasound scans, performed at the end of the first trimester. cUS was obtained on the first, third, and seventh day of life and 
repeated weekly until discharge. aEEG sequences of infants sedated with drugs other than morphine or treated with antiepileptics were excluded from the analysis.

\section{Data Acquisition and Analysis Procedure}

One-channel aEEG was recorded with biparietal hydrogel electrodes, corresponding to $\mathrm{P} 3-\mathrm{P} 4$, according to the international electroencephalogram classification 10-20 system, ground $F_{z}$, with the Brainz Instruments BRM3 monitor (Natus Medical, San Carlos, CA). The technique and the physiological background of the aEEG have been outlined in detail elsewhere (33). aEEG recording started within the first $24 \mathrm{~h}$ after birth and lasted until days 3-4. Each 3-h artifact-free period with impedance of less than $10 \mathrm{kOhm}$ was analyzed. aEEG tracings were rated offline using two different rating methods based on preestablished criteria of visual and mechanical analysis $(10,11)$. aEEG rating was performed by two authors (G.N. and C.H.) blinded toward the subject's identity and clinical course. Results were compared, and if disagreement was present, the rating was discussed and agreed upon. Interrater agreement was expressed with the Cohen's $\kappa$ (see above). Analysis of the aEEG was performed as described in the following sections.

\begin{abstract}
"Maturity total score" and "cycling subscore." Visual analysis of each 3-h period was performed using the scoring system for brain maturity evaluation as described by Burdjalov et al. (11). Four components were graded (for details please see reference): continuity, cycling, amplitude of the lower border, and bandwidth of the aEEG amplitude. Each component was scored and summed to define a "maturity total score," ranging from 0 to 13 with lower scores signifying less-mature brain activity. Because of its apparent role as indicator of brain maturity (11) and its prognostic relevance $(4,5,7)$, the "cycling" component, ranging from 0 to 5, was separately analyzed and called "cycling subscore" $(11,34)$.
\end{abstract}

Mechanical quantitative analysis. Mechanical quantitative analysis of the aEEG tracings was performed using the BrainZ Analyse Research software (Chart analyzer 1.71, The Liggins Institute, Auckland, New Zealand), which exports the raw data and calculates the 1-min average values for the maximum and minimum amplitudes of each 3-h aEEG trace period (10). The maximum and minimum aEEG amplitude corresponds to the upper and lower border of the aEEG envelope, respectively. We measured the mean amplitudes values for each aEEG period instead of measuring it during the quiet and active sleep period separately, because sleep phases are difficult to define in preterm infants.

\section{Statistics}

We assessed the effect of GA, PNA, and other perinatal factors on the various aEEG measurements using regression coefficients in a linear mixed model, calculated with the lme routine from the nlme package available in the free statistical (software R, version 2.5.1 (The R Foundation for Statistical Computing, Vienna, Austria). We performed both univariate analyses, where a single factor was introduced in the model, and a multivariate analysis, with all factors in the model. Covariates included in the analyses were GA, PNA, and perinatal factors suspected to influence aEEG/EEG activity or neurologic outcome according to evidences from the literature $(3,7,9,25,27,34-36)$, including male sex, small-for-GA status (birth weight $<10$ centile), chorioamnionitis (defined by histopathologic examination of the placenta), complete antenatal corticosteroids for lung maturation (37), arterial umbilical cord pH, 5-min Apgar score, the highest decile for GA of the SNAPPE-II (38). Furthermore, perinatal factors (recorded during aEEG monitoring) included mechanical ventilation, surfactant therapy, proven sepsis (positive blood or cerebrospinal fluid culture) (39), hypoglycemia (blood glucose level $<2.5 \mathrm{mmol} / \mathrm{l}$ ), and administration of morphine for sedation, caffeine for apnea therapy, and indomethacin for therapy of hemodynamically relevant patent ductus arteriosus (for dose and therapy duration please see Table 1). To account for repeated aEEG measures, a random "infant effect" was introduced in each model. Whereas the coefficient associated with PNA (the PNA effect) refers to the postnatal development of aEEG, being an estimation of the average daily development during the first $4 \mathrm{~d}$ of life, the coefficient associated with GA (the GA effect) refers to the prenatal development, being an estimation of the average difference of aEEG at birth between two groups of infants differing of $1 \mathrm{~d}$ with respect to GA. This 1-d difference is a statistical estimate, and it does not equal a true 1-d difference in GA, as the accuracy of GA estimation is not precise.

$P$ values $<0.05$ were considered significant and $95 \%$ confidence intervals are provided for each coefficient.

\section{ACKNOWLEDGMENTS}

We gratefully thank all the children and their parents who participated in this study.

\section{STATEMENT OF FINANCIAL SUPPORT}

G.N. was funded by the Swiss National Science Foundation, grant 33CM30124101.

Disclosure: All authors declare no actual or potential conflict of interest.

\section{REFERENCES}

1. Iams JD, Romero R, Culhane JF, Goldenberg RL. Primary, secondary, and tertiary interventions to reduce the morbidity and mortality of preterm birth. Lancet 2008;371:164-75

2. Fanaroff AA, Stoll BJ, Wright LL, et al.; NICHD Neonatal Research Network. Trends in neonatal morbidity and mortality for very low birthweight infants. Am J Obstet Gynecol 2007;196:147.e1-8.

3. Toet MC, Hellström-Westas L, Groenendaal F, Eken P, de Vries LS. Amplitude integrated EEG 3 and 6 hours after birth in full term neonates with hypoxic-ischaemic encephalopathy. Arch Dis Child Fetal Neonatal Ed 1999;81:F19-23.

4. Klebermass K, Olischar M, Waldhoer T, Fuiko R, Pollak A, Weninger M. Amplitude-integrated EEG pattern predicts further outcome in preterm infants. Pediatr Res 2011;70:102-8.

5. Wikström S, Pupp IH, Rosén I, et al. Early single-channel aEEG/ EEG predicts outcome in very preterm infants. Acta Paediatr 2012; 101:719-26.

6. Olischar M, Klebermass K, Kuhle S, et al. Reference values for amplitudeintegrated electroencephalographic activity in preterm infants younger than 30 weeks' gestational age. Pediatrics 2004;113(1 Pt 1):e61-6.

7. Hellström-Westas L, Rosén I, de Vries LS, Greisen G. Amplitude-integrated EEG classification and interpretation in preterm and term infants. Neoreviews 2006; 7:e76-87.

8. Klebermass K, Kuhle S, Olischar M, Rücklinger E, Pollak A, Weninger M. Intra- and extrauterine maturation of amplitude-integrated electroencephalographic activity in preterm infants younger than 30 weeks of gestation. Biol Neonate 2006;89:120-5.

9. Herbertz S, Pulzer F, Gebauer C, Panhofer M, Robel-Tillig E, Knüpfer M. The effect of maturation and sedation on amplitude-integrated electroencephalogram of the preterm neonate: results of a prospective study. Acta Paediatr 2006;95:1394-9.

10. West CR, Harding JE, Williams CE, Gunning MI, Battin MR. Quantitative electroencephalographic patterns in normal preterm infants over the first week after birth. Early Hum Dev 2006;82:43-51.

11. Burdjalov VF, Baumgart S, Spitzer AR. Cerebral function monitoring: a new scoring system for the evaluation of brain maturation in neonates. Pediatrics 2003;112:855-61.

12. Griesmaier E, Enot DP, Bachmann M, et al. Systematic characterization of amplitude-integrated EEG signals for monitoring the preterm brain. Pediatr Res 2013;73:226-35.

13. Sisman J, Campbell DE, Brion LP. Amplitude-integrated EEG in preterm infants: maturation of background pattern and amplitude voltage with postmenstrual age and gestational age. J Perinatol 2005;25:391-6.

14. Soubasi V, Mitsakis K, Nakas CT, et al. The influence of extrauterine life on the aEEG maturation in normal preterm infants. Early Hum Dev 2009;85:761-5. 


\section{Articles $\mid$ Natalucciet al.}

15. Niemarkt HJ, Andriessen P, Peters $\mathrm{CH}$, et al. Quantitative analysis of amplitude-integrated electroencephalogram patterns in stable preterm infants, with normal neurological development at one year. Neonatology 2010;97:175-82.

16. Smith GC, Gutovich J, Smyser C, et al. Neonatal intensive care unit stress is associated with brain development in preterm infants. Ann Neurol 2011;70:541-9.

17. Scher MS. Neurophysiological assessment of brain function and maturation: I. A measure of brain adaptation in high risk infants. Pediatr Neurol 1997;16:191-8.

18. Scher MS. Neurophysiological assessment of brain function and maturation. II. A measure of brain dysmaturity in healthy preterm neonates. Pediatr Neurol 1997;16:287-95.

19. Tombini M, Pasqualetti P, Rizzo C, et al. Extrauterine maturation of somatosensory pathways in preterm infants: a somatosensory evoked potential study. Clin Neurophysiol 2009;120:783-9.

20. Yamamoto N, Watanabe K, Sugiura J, Okada J, Nagae H, Fujimoto Y. Marked latency change of auditory brainstem response in preterm infants in the early postnatal period. Brain Dev 1990;12:766-9.

21. Atkinson J, Anker S, Rae S, Weeks F, Braddick O, Rennie J. Cortical visual evoked potentials in very low birthweight premature infants. Arch Dis Child Fetal Neonatal Ed 2002;86:F28-31.

22. Scher MS, Ludington-Hoe S, Kaffashi F, Johnson MW, Holditch-Davis D, Loparo KA. Neurophysiologic assessment of brain maturation after an 8 -week trial of skin-to-skin contact on preterm infants. Clin Neurophysiol 2009;120:1812-8.

23. Bernet V, Latal B, Natalucci G, Doell C, Ziegler A, Wohlrab G. Effect of sedation and analgesia on postoperative amplitude-integrated EEG in newborn cardiac patients. Pediatr Res 2010;67:650-5.

24. Bell AH, Greisen G, Pryds O. Comparison of the effects of phenobarbitone and morphine administration on EEG activity in preterm babies. Acta Paediatr 1993;82:35-9.

25. Supcun S, Kutz P, Pielemeier W, Roll C. Caffeine increases cerebral cortical activity in preterm infants. J Pediatr 2010;156:490-1.

26. Itakura A, Kurauchi O, Morikawa S, Matsuzawa K, Mizutani S, Tomoda Y. [Neonatal EEG findings soon after birth in the intrauterine growth retarded infant]. Nippon Sanka Fujinka Gakkai Zasshi 1995;47:109-14.
27. Wikström S, Ley D, Hansen-Pupp I, Rosén I, Hellström-Westas L. Early amplitude-integrated EEG correlates with cord TNF-alpha and brain injury in very preterm infants. Acta Paediatr 2008;97:915-9.

28. Keogh MJ, Bennet L, Drury PP, et al. Subclinical exposure to low-dose endotoxin impairs EEG maturation in preterm fetal sheep. Am J Physiol Regul Integr Comp Physiol 2012;303:R270-8.

29. Dempsey EM, Barrington KJ. Diagnostic criteria and therapeutic interventions for the hypotensive very low birth weight infant. J Perinatol 2006;26:677-81.

30. Volpe JJ. Intracranial hemorrhage: germinal matrix-intraventricular hemorrhage. In: Volpe JJ, ed. Neurology of the Newborn. Philadelphia, PA: Saunders Elsevier, 2008:517.

31. de Vries LS, Eken P, Dubowitz LM. The spectrum of leukomalacia using cranial ultrasound. Behav Brain Res 1992;49:1-6.

32. Levene MI. Measurement of the growth of the lateral ventricles in preterm infants with real-time ultrasound. Arch Dis Child 1981; 56:900-4.

33. Rosén I. The physiological basis for continuous electroencephalogram monitoring in the neonate. Clin Perinatol 2006;33:593-611, v.

34. Natalucci G, Rousson V, Bucher HU, Bernet V, Hagmann C, Latal B. Delayed cyclic activity development on early amplitudeintegrated EEG in the preterm infant with brain lesions. Neonatology 2013;103:134-40.

35. Flisberg A, Kjellmer I, Löfhede J, et al. Does indomethacin for closure of patent ductus arteriosus affect cerebral function? Acta Paediatr 2010;99:1493-7.

36. Lee HJ, Kim HS, Kim SY, et al. Effects of postnatal age and aminophylline on the maturation of amplitude-integrated electroencephalography activity in preterm infants. Neonatology 2010;98:245-53.

37. Baud O, Foix-L'Helias L, Kaminski M, et al. Antenatal glucocorticoid treatment and cystic periventricular leukomalacia in very premature infants. $\mathrm{N}$ Engl J Med 1999;341:1190-6.

38. Richardson DK, Corcoran JD, Escobar GJ, Lee SK. SNAP-II and SNAPPEII: simplified newborn illness severity and mortality risk scores. J Pediatr 2001;138:92-100.

39. Schlapbach LJ, Aebischer M, Adams M, et al.; Swiss Neonatal Network and Follow-Up Group. Impact of sepsis on neurodevelopmental outcome in a Swiss National Cohort of extremely premature infants. Pediatrics 2011;128:e348-57. 